\title{
The fortedata $\mathbf{R}$ package: open-science datasets from a manipulative experiment testing forest resilience
}

5 Jeff W. Atkins ${ }^{1}$, Elizabeth Agee ${ }^{2}$, Alexandra Barry ${ }^{1}$, Kyla M. Dahlin ${ }^{3}$, Kalyn Dorheim ${ }^{4}$, Maxim S. Grigri ${ }^{1}$, Lisa T. Haber ${ }^{1}$, Laura J. Hickey ${ }^{1}$, Aaron G. Kamoske ${ }^{3}$, Kayla Mathes ${ }^{1}$, Catherine McGuigan ${ }^{1}$, Evan Paris ${ }^{5}$, Stephanie C. Pennington ${ }^{4}$, Carly Rodriguez ${ }^{6}$, Autym Shafer ${ }^{1}$, Alexey Shiklomanov ${ }^{7}$, Jason Tallant ${ }^{8}$, Christopher M. Gough ${ }^{1}$, Ben Bond-Lamberty ${ }^{4}$

$10 \quad{ }^{1}$ Department of Biology, Virginia Commonwealth University, Richmond, VA 23059, USA

${ }^{2}$ Environmental Sciences Division and Climate Change Science Institute, Oak Ridge National Laboratory, Oak Ridge, TN, 37831, USA

${ }^{3}$ Department of Geography, Environment, \& Spatial Sciences, Michigan State University, East Lansing, MI 48824, USA

${ }^{4}$ Joint Global Change Research Institute at the University of Maryland, Pacific Northwest National Laboratory, College Park,

15 MD, 20740, USA

${ }^{5}$ Vassar College, Poughkeepsie, NY, 12604, USA

${ }^{6}$ Western Colorado University, Gunnison, CO, 81231, USA

${ }^{7}$ NASA Goddard Space Flight Center, Greenbelt, MD 20771, USA

${ }^{8}$ University of Michigan Biological Station, Pellston, MI, 49769, USA

Correspondence to: Jeff W. Atkins (jwatkins6@vcu.edu)

Plots table

Table S1. Plots table. The fd_plots ( ) function is an internal function that contains plot level metadata.

\begin{tabular}{llll} 
field & description & class & units \\
\hline replicate & Replicate group (A-D) & character & \\
plot & Plot number & integer & \\
& & & \\
latitude_plot & Decimal latitude of plot center & numeric & degrees N \\
longitude_plot & Decimal longitude of plot center & numeric & degrees E \\
plot_area_m2 & Plot area & integer & $\mathrm{m} 2$
\end{tabular}

25 Subplots table

Table S2. Subplots table. fd_subplots ( ) is an internal function that returns subplot-level metadata.

field description

class units 


$\begin{array}{llll}\text { replicate } & \text { Replicate group (A-D) } & \text { character } \\ \text { plot } & \text { Plot number } & \text { integer } & \\ \text { subplot } & \text { Subplot }(\mathrm{E}, \mathrm{W}) & \text { character } & \\ & & & \\ \text { latitude_subplot } & \text { Decimal latitude of subplot center } & \text { numeric } & \text { degrees N } \\ \text { longitude_subplot } & \text { Decimal longitude of subplot center } & \text { numeric } & \text { degrees E } \\ \text { subplot_area_m2 } & & \text { integer } & \text { m2 }\end{array}$

\section{Nested subplots table}

Table S3. Nested subplots table. fd_nested_subplots ( ) is an internal function that returns nested subplot metadata. Nested subplots are either sampling points $(0,1,3,5,7)$ for specific FoRTE

30 measurements (e.g. soil respiration, micrometeorology) or $4 \mathrm{~m} 2$ herbaceous layer vegetation sampling plots $(2,4,6,8)$-illustrated in Figure $1 B$.

\begin{tabular}{|c|c|c|c|}
\hline field & description & class & units \\
\hline replicate & Replicate group (A-D) & character & \\
\hline plot & Plot number & integer & \\
\hline subplot & Subplot $(E, W)$ & character & \\
\hline nested_subplot & Nested subplot number & integer & \\
\hline nested_subplot_area_m2 & Nested subplot area & integer & $\mathrm{m} 2$ \\
\hline
\end{tabular}

Number of Observations

Inventory dataset

35 Table S4. Inventory dataset. fd_inventory is an external function that returns forest inventory data.

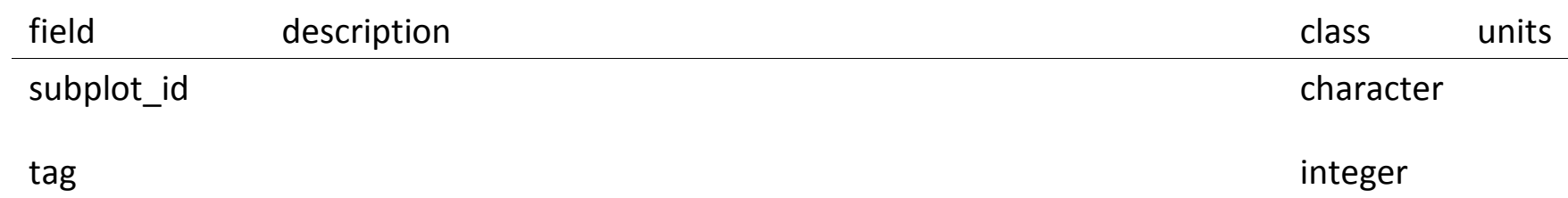




\begin{tabular}{|c|c|c|}
\hline species & Species code from the USDA Plants Database & character \\
\hline dbh_cm & Bole diameter at $1.37 \mathrm{~m}$ & numeric \\
\hline health_status & Live (L), moribund (M), or dead (D) & character \\
\hline canopy_status & $\begin{array}{l}\text { Overstory dominant (OD), overstory submissive (OS), sapling } \\
(\mathrm{SA}) \text {, understory (UN) }\end{array}$ & character \\
\hline date & Date of measurement & date \\
\hline notes & & character \\
\hline replicate & (from plots table) & character \\
\hline plot & (from plots table) & integer \\
\hline subplot & (from subplots table) & character \\
\hline
\end{tabular}

\section{Soil respiration}

Table S5. Soil respiration table. fd_soil_respiration( ) is an external function that returns soil respiration collected with a LI-COR Biosciences LI-6400 portable gas analyzer with $10 \mathrm{~cm}$ diameter soil CO2 closed chamber cuvette (LI-COR Inc, Lincoln, NE, USA). This dataset includes concurrently 40 measured soil temperature (from surface to $7 \mathrm{~cm}$ depth) and soil water content (measured using a Campbell Sciences CS620 (Logan, UT).

\begin{tabular}{|c|c|c|}
\hline field & description & units \\
\hline subplot_id & $\begin{array}{l}\text { Subplot ID number, a concatenation of replicate, plot, } \\
\text { and subplot codes }\end{array}$ & character \\
\hline replicate & Replicate group (A-D) & character \\
\hline plot & Plot number & integer \\
\hline subplot & Subplot $(E, W)$ & character \\
\hline date & Date of measurement & date \\
\hline timestamp & Timestamp of measurement & POSIXct \\
\hline nested_subplot & Nested subplot sampling point inside subplot & character \\
\hline
\end{tabular}


run

integer

soil_co2_efflux Soil surface CO2 efflux measured with a LI-6400

numeric $\hat{A} \mu \mathrm{mol}$

soil_temp

Soil temperature measured at $7 \mathrm{~cm}$ depth

numeric degrees $\mathrm{C}$

vWC

Volumetric water content

numeric percent

\section{Leaf spectrometry}

Table S6. Leaf spectrometry. fd_leaf_spectrometry ( ) is an external function that returns 45 vegetation indices calculated from leaf spectra data collected on leaves from understory vegetation with a $\mathrm{CID}$ Biosciences $\mathrm{Cl}-710$ Handheld spectrometer (Camas, WA).

\begin{tabular}{|c|c|c|}
\hline field & description & class \\
\hline subplot_id & $\begin{array}{l}\text { Subplot ID number, a concatenation of replicate, plot, and subplot } \\
\text { codes }\end{array}$ & character \\
\hline replicate & Replicate group (A-D) & character \\
\hline plot & Plot number & integer \\
\hline subplot & Subplot $(E, W)$ & character \\
\hline date & Date of measurement & Date \\
\hline species & $\begin{array}{l}\text { Species code from the USDA Plants Database; see } \\
\text { https://plants.sc.egov.usda.gov/java/ }\end{array}$ & character \\
\hline index & Spectral index measured from the CID 710 & character \\
\hline index_value & Measured index value corresponding to the index & numeric \\
\hline
\end{tabular}

\section{Photosynthesis}

Table S7. Photosynthesis table. fd_photosynthesis ( ) is an external function that returns ecophysiological data (e.g. photosynthesis, transpiration) on subcanopy leaves measured with a LICOR Biosciences LI-6400 (Lincoln, NE).

field description

class units 


\begin{tabular}{|c|c|c|c|}
\hline subplot_id & $\begin{array}{l}\text { Subplot ID number, a concatenation of replicate, plot, and } \\
\text { subplot codes }\end{array}$ & character & \\
\hline replicate & Replicate group (A-D) & character & \\
\hline plot & Plot number & integer & \\
\hline subplot & Subplot $(E, W)$ & character & \\
\hline timestamp & Timestamp of measurement & POSIXct & \\
\hline obs & Observation number within file & integer & \\
\hline ftime & Number of seconds since logging began & numeric & \\
\hline ebal & Energy balance on? & integer & \\
\hline photo & Photosynthetic rate & numeric & $\begin{array}{l}\mu \mathrm{mol} \\
\mathrm{CO} 2 / \mathrm{m} 2 / \mathrm{s}\end{array}$ \\
\hline cond & Stomatal conductance & numeric & $\begin{array}{l}\mathrm{mol} \\
\mathrm{H} 2 \mathrm{O} / \mathrm{m} 2 / \mathrm{s}\end{array}$ \\
\hline ci & Intracellular $\mathrm{CO} 2$ concentration & numeric & $\mu \mathrm{mol} / \mathrm{mol}$ \\
\hline trmmol & Transpiration rate & numeric & $\begin{array}{l}\mathrm{mmol} \\
\mathrm{H} 2 \mathrm{o} / \mathrm{m} 2 / \mathrm{s}\end{array}$ \\
\hline vpdl & Leaf-level vapor pressure deficit & numeric & $\mathrm{kPa}$ \\
\hline ctleaf & Leaf temperature from energy balance calculation & numeric & degrees C \\
\hline area & $\begin{array}{l}\text { In-chamber leaf area (note that this area is not the true } \\
\text { sampled tissue area for needleleaf samples) }\end{array}$ & numeric & $\mathrm{cm} 2$ \\
\hline blc_1 & One sided boundary layer conductance & numeric & $\mathrm{mol} / \mathrm{m} 2 / \mathrm{s}$ \\
\hline stmrat & Stomatal ratio estimate & numeric & \\
\hline blcond & Boundary layer conductance & numeric & $\mathrm{mol} / \mathrm{m} 2 / \mathrm{s}$ \\
\hline tair & Chamber air temperature & numeric & degrees C \\
\hline tleaf & Leaf surface temperature & numeric & degrees C \\
\hline tblk & IRGA block temperature & numeric & degrees C \\
\hline $\operatorname{co} 2 r$ & Reference $\mathrm{CO} 2$ concentration & numeric & $\mu \mathrm{mol} / \mathrm{mol}$ \\
\hline $\operatorname{co} 2 \mathrm{~s}$ & Sample CO2 concentration & numeric & $\mu \mathrm{mol} / \mathrm{mol}$ \\
\hline h2or & Reference $\mathrm{H} 20$ concentration & numeric & $\mu \mathrm{mol} / \mathrm{mol}$ \\
\hline
\end{tabular}




\begin{tabular}{|c|c|c|c|}
\hline h2os & Sample H2O concentration & numeric & $\mu \mathrm{mol} / \mathrm{mol}$ \\
\hline$r h \_r$ & Reference relative humidity & numeric & percent \\
\hline rh_s & Sample relative humidity & numeric & percent \\
\hline flow & Flow rate & numeric & $\mu \mathrm{mol} / \mathrm{mol}$ \\
\hline pari & In-chamber PAR & integer & $\mu \mathrm{mol} / \mathrm{m} 2 / \mathrm{s}$ \\
\hline paro & External PAR & integer & $\mu \mathrm{mol} / \mathrm{m} 2 / \mathrm{s}$ \\
\hline press & Atmospheric pressure & numeric & $\mathrm{kPa}$ \\
\hline csmch & & numeric & \\
\hline hsmch & & numeric & \\
\hline csmchsd & $\begin{array}{l}\text { Standard deviation of } \mathrm{CO} 2 \mathrm{~S} \text { during averaging time of most } \\
\text { recent match }\end{array}$ & numeric & \\
\hline hsmchsd & $\begin{array}{l}\text { Standard deviation of } \mathrm{H} 2 \mathrm{OS} \text { during averaging time of most } \\
\text { recent match }\end{array}$ & numeric & \\
\hline crmchsd & $\begin{array}{l}\text { Standard deviation of } \mathrm{CO} 2 \mathrm{~S} \text { during averaging time of most } \\
\text { recent match }\end{array}$ & numeric & \\
\hline hrmchsd & $\begin{array}{l}\text { Standard deviation of } \mathrm{CO} 2 \mathrm{R} \text { during averaging time of most } \\
\text { recent match }\end{array}$ & numeric & \\
\hline stablef & Stable/total as a fraction & numeric & \\
\hline blcslope & Slope term used in calculating boundary layer conductance & numeric & \\
\hline blcoffst & $\begin{array}{l}\text { Intercept term using in calculating boundary layer } \\
\text { conductance }\end{array}$ & numeric & \\
\hline f_parin & Fraction of Parln_um to use for energy balance & numeric & \\
\hline f_parout & Fraction of ParOut_um to use for energy balance & numeric & \\
\hline alphak & Used in conversion of $\hat{A} \mu \mathrm{mol} / \mathrm{mol}$ to $\mathrm{W} / \mathrm{m} 2$ & numeric & \\
\hline status & Status variable & integer & \\
\hline species & $\begin{array}{l}\text { Species code from the USDA Plants Database; see } \\
\text { https://plants.sc.egov.usda.gov/java/ }\end{array}$ & character & \\
\hline sample & Sample number varies by species by plot & integer & \\
\hline
\end{tabular}


comments ID of closest vegetation survey plot (NE, SE, SW, NW) to the character stem measured, plus any additional comments

\section{Litter}

Table S8. Litterfall table. fd_litter ( ) is an external function that returns dry litter mass collected in litter traps located at nested subplots $(1,3,5,7)$ within each FoRTE subplot. The bag_mass_g values represent air-dried mass.

\begin{tabular}{|c|c|c|c|}
\hline field & description & class & units \\
\hline subplot_id & $\begin{array}{l}\text { Subplot ID number, a concatenation of replicate, plot, and subplot } \\
\text { codes }\end{array}$ & character & \\
\hline replicate & Replicate group (A-D) & character & \\
\hline plot & Plot number & integer & \\
\hline subplot & Subplot $(E, W)$ & character & \\
\hline year & Year of litter collection & integer & \\
\hline species & $\begin{array}{l}\text { Species code from the USDA Plants Database; see } \\
\text { https://plants.sc.egov.usda.gov/java/ }\end{array}$ & character & \\
\hline bagtare_g & Mass of bag & numeric & g \\
\hline bagmass_g & Mass of bag + litter & numeric & g \\
\hline
\end{tabular}

55 Hemispherical camera

Table S9. Hemispherical imagery table. fd_hemi_camera ( ) is an external function that returns undercanopy, hemispherical imagery captured using a Sony DSLR camera (Regent Instruments; Quebec, QU) where the blue channel has been replaced with near-infrared in order to calculate normalized difference vegetation index (NDVI). Raw imagery has been processed using WinsCANOPY

60 (Regent Instruments; Quebec, QU).

\begin{tabular}{|c|c|c|}
\hline field & description & class \\
\hline subplot_id & $\begin{array}{l}\text { Subplot ID number, a concatenation of replicate, plot, and } \\
\text { subplot codes }\end{array}$ & character \\
\hline replicate & Replicate group (A-D) & character \\
\hline plot & Plot number & integer \\
\hline
\end{tabular}




$\begin{array}{llr}\text { subplot } & \text { Subplot }(\mathrm{E}, \mathrm{W}) & \text { character } \\ \text { nested_subplot } & \text { NestedSubplotSampling points } & \text { integer } \\ \text { date } & \text { Date of measurement } & \text { Date } \\ \text { ndvi } & \text { Normalized Difference Vegetation Index, estimates greenness } & \text { numeric } \\ \text { gap_fraction } & \text { Ratio of gap space in the canopy, or open area } & \text { numeric } \\ \text { openness } & \text { Fraction of open gap space in the canopy } & \text { numeric } \\ \text { lai_cam } & & \text { Leaf area index } \\ \text { clumping_index } & \text { The ratio of the effective leaf area index to the true leaf area } & \text { numeric } \\ & \text { index }\end{array}$

\section{Canopy structure}

Table S10. Canopy structural traits table. fd_canopy_structure ( ) is an external function that returns canopy structural trait data collected using a terrestrial based portable canopy lidar unit (Regent Instruments 3100 FHS; . Raw lidar data has been processed using forestr version 1.0.1 65 (Atkins et al. 2018) in R 3.6 (R Core Team, 2020) to calculate canopy structural trait metrics.

\begin{tabular}{|c|c|c|}
\hline field & description & class \\
\hline subplot_id & $\begin{array}{l}\text { Subplot ID number, a concatenation of replicate, plot, and } \\
\text { subplot codes }\end{array}$ & character \\
\hline replicate & Replicate group (A-D) & character \\
\hline plot & Plot number & integer \\
\hline subplot & Subplot $(E, W)$ & character \\
\hline year & Year of measurement & integer \\
\hline mean.height & Mean height of vai & numeric \\
\hline height.2 & Standard deviation of mean height & numeric \\
\hline mean.height.var & Variance of mean height & numeric \\
\hline
\end{tabular}




\begin{tabular}{|c|c|c|}
\hline mean.height.rms & Root mean square height & numeric \\
\hline transect.length & Length of transect & integer \\
\hline mode.el & Mean height of maximum VAI & numeric \\
\hline max.el & Greatest density of vai x, z position & numeric \\
\hline mode.2 & Variance of maximum vai & numeric \\
\hline max.can.ht & Maximum measured canopy height & numeric \\
\hline mean.max.ht & Mean outer canopy height or moch & numeric \\
\hline mean.vai & Average vai across transect & numeric \\
\hline mean.peak.vai & Average height of maximum vai & numeric \\
\hline deep.gaps & Number of $1 \mathrm{~m}$ wide bins with no lidar returns & integer \\
\hline deep.gap.fraction & Deep gaps dividied by transect length & numeric \\
\hline porosity & Ratio of empty to filled bins in the canopy & numeric \\
\hline std.std & Precursor to rugosity & numeric \\
\hline mean.std & Precursor to rugosity & numeric \\
\hline rugosity & Accumulated canopy complexity metric & numeric \\
\hline top.rugosity & Standard deviation of final lidar returns & numeric \\
\hline mean.return.ht & Average lidar return distance & numeric \\
\hline sd.return.ht & Standard deviation of lidar return distances & numeric \\
\hline sky.fraction & Ratio of sky hits to lidar returns & numeric \\
\hline cover.fraction & $1 /$ sky fraction & numeric \\
\hline max.ht & Same as max can ht, removed in later forestr updates & numeric \\
\hline
\end{tabular}


scan.density

rumple

clumping.index

enl

index

numeric

Effective number of layers

numeric

\section{Ceptometer}

Table S11. Ceptometer table. fd_par ( ) is an external function that returns concurrently measured above- and below-canopy light availability, as well as leaf area index (calculated from above- and below-canopy light) collected along $40 \mathrm{~m}$ long north-to-south and east-to-west transects through the 70 center of each FoRTE subplot. Data were collected using a Decagon Devices LP-80 handheld ceptometer (Pullman, WA).

\begin{tabular}{|c|c|c|}
\hline field & description & class \\
\hline subplot_id & $\begin{array}{l}\text { Subplot ID number, a concatenation of replicate, plot, and subplot } \\
\text { codes }\end{array}$ & character \\
\hline replicate & Replicate group (A-D) & character \\
\hline plot & Plot number & integer \\
\hline subplot & Subplot $(E, W)$ & character \\
\hline timestamp & Timestamp of measurement & POSIXct \\
\hline a_par & Above canopy PAR (photosynthetically available radiation) & numeric \\
\hline b_par & Below canopy PAR (photosynthetically available radiation) & numeric \\
\hline fapar & Fraction of PAR absorbed by the canopy & numeric \\
\hline lai_cept & Leaf area index derived from ceptometer & numeric \\
\hline
\end{tabular}

